\title{
Levetiracetum Induced Angioedema without Prior Reaction to Phenytoin
}

\author{
Dr. Priyajyoti Chakma ${ }^{1}$, Dr. Bappaditya Roy ${ }^{2}$, Dr. Punyadhar Das ${ }^{3}$
}

${ }^{1}$ Registrar, Department of Psychiatry, Agartala Govt. Medical College, Agartala, Tripura West, 799006, India ${ }^{2}$ Post graduate trainee, Department of Psychiatry, Agartala Govt. Medical College, Agartala Tripura West, 799006, India

${ }^{3}$ Prof. \& HOD, Department of Psychiatry, Agartala Govt. Medical College, Agartala

Tripura West, 799006, India

\section{ABSTRACT}

Allergic reactions to antiepileptic drugs in the form of skin rash are not uncommon but angioedema, an acute life threatening reaction is rare. Levetiracetum has been considered relatively safe compared with other anti epileptic drugs with regard to skin reactions. Though very uncommon but angioedema, a life threatening reaction has been reported with carbamazepine and oxcarbamazepine. We report a case of an 18-year-old woman with generalized tonic clonic seizure who developed angioedema following levetiracetam monotherapy. She was previously on phenytoin and seizure attack remained unchanged, levetiracetam was slowly substituted for phenytoin. After the first dose of levetiracetam she developed a generalized maculopapular skin rash with associated swelling of the face, lips and tongue. The patient had no previous history of skin rashes with any drugs. Levetiracetam was stopped and she improved after treatment with, antihistamines and corticosteroids.

Key words : Levetiracetum, cutaneous angioedema, skin rash, seizure

\section{INTRODUCTION}

Hypersensitivity to anti epileptic drugs is unpredictable and not dose related. Amongst different hypersensitivity reaction reported most common type is mild skin rash. ${ }^{1}$ Most serious reaction occurs as toxic epidermal necrolysis, Steven Johnson syndrome or drug reaction with eosinophilia and systemic symptoms. Another

\section{Correspondence :}

Dr Priyajyoti Chakma, Registrar, Department of Psychiatry, AGMC \& GBP Hospital, Agartala 799006, Tripura, India

Phone : 8131043421

Email : pjchakma84@gmail.com uncommon acute life threatening allergic reaction is angioedema. Angioedema is an uncommon acute life threatening allergic reaction to Levetriacetum. Type IV hypersensitivity reaction is considered to be $T$ cell mediated in which antigen binds covalently to a MHC complex on the antigen presenting cells(APC)and subsequently triggering a delayed hypersensitivity reaction. Another mechanism may involve antigen/T cell/MHC interaction through non-covalent binding.1 Recent studies have shown that most anti epileptic drugs (phenytoin, carbamazepine, valproic acid and lamotrigine) related hypersensitivity reactions are secondary to MHC (Major Histocompatibility Complex) dependent clonal T cell proliferation., ${ }^{2,9}$ Type I, II, III reactions are B cell mediated and Type IV involves 
deposition of immune complexes on different tissues causing vasculitis and tissue damage. ${ }^{2}$

We report a case of women who developed angioedema related to levetiracetum monotherapy first dose.

\section{CASE}

18 years old right handed, Hindu, married, literate, woman of middle socio-economic status from rural part of Tripura, who reported in Psychiatric Outpatient Department (OPD) of Agartala Govt. Medical College \& GBP Hospital, Tripura,India who had a generalized tonic clonic seizure which started at the age of 17 years. She then continued to have seizures twice per month. Seizure episode started during sleep with sudden onset of tonic clonic contraction of the both upper and lower limbs with clinching of teeth. Seizure episodes lasted for 5 to 7 minutes followed by loss of consciousness. After gaining consciousness she was amnestic about seizure episode. She was admitted in female psychiatry ward. There was no history of febrile seizure, CNS infection or head trauma. Her neurological examination was unremarkable. Her mental status examination showed depressed affect apart from that no abnormality detected. EEG tracing was done and abnormal electrical activity was found. MRI revealed normal study. She was initially treated with Phenytoin $300 \mathrm{mg}$ and she was maintained on it for one year without any skin rashes or any side effects. Since her seizure activity again started while on medications, Levetiracetam was slowly substituted for phenytoin. Her total body weight was $50 \mathrm{~kg}$. She was placed on levetiracetum $500 \mathrm{mg}$ daily as monotherapy. She was on no other medications and had been off phenytoin for one week. Only after the first dose of Levetriacetum she developed generalized wheal, flare with popular eruption of face and skin with erythema and swelling of face, ear lobules and also both upper limbs. Dermatological consultation was done and diagnosis of urticarial angioedema was made. Her blood pressure and oxygen saturation remained normal. $\mathrm{CBC}$ including eosinophils immunology remained normal. Levetiracetam was discontinued and she was treated with methyl-prednisolone and antihistaminic. Swelling of her lips and tongue resolved within 48 hours and skin rashes disappeared within 5 days. Tablet Clobazam $5 \mathrm{mg}$ thrice daily dose was started, which she tolerated well with no major side effects and remained seizure free.

\section{DISCUSSION}

$3-15 \%$ incidence of skin rashes develops as adverse reaction to anti epileptic drugs. ${ }^{1,3}$ Aromatic compounds such as Phenobarbital, Phenytoin and Carbamazepine has higher risk for developing skin reactions. Lamotrigine, a non-aromatic anti epileptic drug is also associated with high frequency of skin rash if it is introduced rapidly. ${ }^{4}$ Levetiracetam is a broad spectrum anti epileptic that has been shown to be effective for a variety of seizures in adults and children. ${ }^{5}$ Levetiracetam is considered to be effective, well tolerated and safe in patients with epilepsy and other medical conditions that are difficult to manage in view of serious adverse effects occurring with aromatic anticonvulsant group of drugs. A recent case report of dose related drug eruption to Levetiracetam has been described. ${ }^{8}$

The spectrum of allergic reaction varies from simple skin rash to more severe form of Steven Johnson syndrome, toxic epidermal necrolysis or DRESS syndrome. ${ }^{6}$

Angioedema is an allergic reaction similar in appearance to urticaria in which patients develop localized swelling in the dermis and submucosa. ${ }^{7}$ It can be life threatening due to airway obstruction from laryngeal oedema. In this case angioedema developed after Levetiracetam $500 \mathrm{mg}$ single dose. Previously (2-3) cases are reported with levetiracetum induced angioedema but with previous history of skin rashes with aromatic antiepileptic drugs (such as phenytoin).

Conclusion : Although aromatic antiepileptic drugs are known to cause allergic reactions and newer antiepileptic drugs such as levetiracetam are 


\section{Levetiracetum Induced Angioedema without Prior Reaction to Phenytoin}

considered to be safe, they still should be used with caution and with careful monitoring.

\section{REFERENCES}

1. Zaccara G, Fraciotta D, Perucca E. Idiosyncratic adverse reaction to antiepileptic drugs. Epilepsia 2007; 48 : 1223-44.

2. Krauss G. Current understanding of delayed anticonvulsant hypersensitivity reactions. Epilepsy Curr 2006; $6: 33-7$.

3. Arif H, Buchsbaum R, Weintraub D, Koyfman S, SalasHumara C, Bazil CW, et al. Comparison and predictors of rash associated with 15 antiepileptic drugs. Neurology $2007 ; 68$ : 1701-9.

4. Wong IC, Lahtoo SD. Adverse reactions to new anticonvulsant drugs. Drug Saf 2000; $23: 35-56$.
5. Crepeau AZ, Treiman DM. Levetiracetum A comprehensive review. Expert Rev Neurother 2010; 10 : 159-71

6. Kleier RS, Breneman DL, Boiko S. Generalized pustulation as a manifestation of the anticonvulsant hypersensitivity syndrome. Arch Dermatol 1991; 127 : 1361- 4.

7. Temino VM, Peebles Jr RS. The spectrum and treatment of angioedema. Am J Med 2008; 121 : 282-6

8. Beswick TC, Cohen JB. Dose related levetiracetam induced reticulated drug eruption. J Drugs Dermatol 2010; 9 : 409-10.

9. Elias A, Madhusoodanan S, Pudkkadan D, Antony JT. Angioedema and maculopapular eruptions associated with carbamazepine administration. CNS Spectr 2006; 11 : 352-4 